\title{
ADAPTATION AND SERIAL PASSAGE OF BOVINE CORONAVIRUS IN AN ESTABLISHED DIPLOID SWINE TESTICULAR CELL LINE AND SUBSEQUENT DEVELOPMENT OF A MODIFIED LIVE VACCINE
}

\author{
Mark W. Welter \\ Oragen L.C. \\ Des Moines, Iowa 50322
}

\begin{abstract}
A virulent bovine coronavirus isolate (newborn calf diarrheal) was adapted and serially passaged in an established diploid swine testicular cell line (ST cells). The same cells have been used to produce modified live porcine rotavirus and coronavirus vaccines that are federally licensed and sold worldwide. Growth of the bovine coronavirus resulted in cytopathic effect characterized by cellular stranding and subsequent cell lysis. Virus yields were relatively high in the ST cells and active replication was confirmed by immune electron microscopy and immunofluorescence. Adaptation of bovine coronavirus to a diploid swine cell line has not been previously reported.

Different cell culture passage levels of bovine coronavirus were evaluated by oral inoculation of clean-catch, colostrum-deprived calves. A passage level of bovine coronavirus was identified that multiplied in the calf without the clinical signs of disease associated with virulent passages. The modified live bovine coronavirus vaccine remained safe and efficacious even after 5-backpassages in calves. Further efficacy studies have shown that the modified live bovine coronavirus vaccine significantly protected calves from highly virulent challenges with either winter dysentery or newborn calf diarrheal coronavirus isolates.
\end{abstract}

\section{INTRODUCTION}

Coronaviruses are a major cause of gastroenteritis in newborn calves and adult cattle worldwide. It has been estimated that economic losses due to neonatal diarrhea approach 
$\$ 500$ million annually for calves in North America alone (Saif and Theil, 1990). On a worldwide basis, this translates into significant economic losses in animal production. Although commercial vaccines are available their efficacy is limited in both experimental and field conditions. This is directly attributed to the strain or biotype of coronavirus used in the vaccines.

For years it had been assumed that all coronavirus infections in cattle were the same, but it has now been shown that two biotypes of bovine coronavirus exist (Millane, 1995). Winter dysentery (WD) is one type of disease due to bovine coronavirus infection. This disease is caused by pneumoenteric bovine coronaviruses that replicate in the epithelium of the upper respiratory tract and the enterocytes of the intestinal tract. The acute disease in adult cattle has been characterized by dark bloody watery diarrhea. These clinical outbreaks frequently result in depression and dehydration which can lead to a decreased milk production. Winter dysentery spreads rapidly to cattle of all ages with infections in young calves resulting in clinical diarrhea and frequently rhinitis (Saif and Theil, 1990). Winter dysentery infections result in high morbidity but low mortality.

Another type of disease due to bovine coronavirus infection has been described as newborn calf diarrheal (NCD). These coronaviruses infect the differentiating epithelial cells of the small intestine and colon (Saif and Theil, 1990). Virus infections in young calves result in villous atrophy leading to watery diarrhea with high morbidity and moderate mortality rates. Researchers have shown that both of these coronavirus biotypes can be differentiated by hemagglutination assays performed at $4^{\circ} \mathrm{C}$ and $37^{\circ} \mathrm{C}$ (Millane, 1995).

Recent work has shown that animals that have recovered from an acute infection of WD are susceptible to a subsequent challenge with NCD. Animals that have recovered from an acute infection with NCD are not susceptible and are completely immune to subsequent challenges with WD. The current vaccines on the market have been reported to have limited field efficacy and it has been suggested that the strain of virus used in the products will not sufficiently protect against all bovine coronavirus infections.

A bovine coronavirus vaccine has been developed and is briefly described in this presentation. The vaccine contains a NCD strain of bovine coronavirus and studies have confirmed that this vaccine will prevent bovine coronavirus diseases due to either WD or NCD infections. The modified live vaccine described in this paper remained safe and efficacious even after 5-backpassages in clean-catch, colostrum-deprived calves. This new and unique vaccine will provide the worldwide cattle industry with a safe and most effective means of controlling bovine coronavirus infections.

\section{METHODS AND RESULTS}

\section{Growth of BCV (NCD Strain) in STs}

A field isolate of bovine coronavirus (NCD strain) was adapted and serially passaged in an established diploid swine testicular cell line (ST cells). Virus infectivity is best described as a typical cytopathic effect (CPE) resulting in a moderate fusogenic characteristic leading to cellular stranding and complete death within 2 days after inoculation. This cytopathology was compared to the reference Mebus strain and found to be characteristically different with the Mebus strain of BCV being very weakly fusogenic. Active replication and identity of the BCV (NCD strain) were confirmed by immunofluorescence, immune electron microscopy and differential hemeagglutination assays. Virus yields are 
relatively high with titers ranging from $10^{8}$ to $10^{9} \mathrm{TCID}_{50} / \mathrm{ml}$. The ST cell line that was used is a federally licensed cell line that has been used to produce the modified live porcine rotavirus and coronavirus vaccines that are sold worldwide. This ST cell line is different than other ST cell lines that are commercially available. The diploid chromosome number of this ST cell line has been shown to remain stabile even after 40 passages from master stock. Unlike the commercially available ST cells which are at a much higher passage level and are aneuploidy.

\section{Selection of a BCV Vaccinal Candidate and Preparation of Master Seed}

Development of a modified live bovine coronavirus (NCD strain) vaccine was achieved by serial passage of the virus in ST cells. All vaccine safety evaluations were conducted in clean catch, colostrum-deprived calves (cc-calves). These calves were housed in germfree bubbles and fed a medicated milk replacer that was previously determined to be devoid of BCV antibodies. This animal model was used to conduct the differential virulence studies since cc-calves are the most susceptible and thereby the most sensitive model available. Different passage levels of BCV were used to orally inoculate cc-calves. The BCV cell culture passages were classified into three categories based on their infectivity for the cc-calves. The first category was defined as those BCV cell culture passage that were still virulent when inoculated into cc-calves as determined by clinical observations. The second category was defined as those BCV cell culture passages that were not virulent for cc-calves yet still infectious as measured by virus shedding and seroconversion. The third category was defined as those BCV cell culture passages that did not replicate in cc-calves and were therefore considered to be over-attenuated. After these studies were completed a BCV (NCD) cell culture passage level was identified and labeled as a vaccine candidate. The virus was then plaque purified three times and a large pool of virus was generated and designated as master seed virus (MSV). The master seed has passed all purity, identity and safety tests as outlined by 9CFR guidelines including extensive adventitious virus tests.

\section{Backpassage of BCV (NCD) in Clean Catch Calves}

The reversion to virulence study was conducted using the BCV MSV as the original inoculum. Two cc-calves were used for initial inoculation and one cc-calf for each subsequent backpassage of the BCV vaccinal strain. All calves were orally inoculated between 5 and 7 days of age. Animals were sacrificed and samples of duodenum, jejunum and ileum were collected for gross histopathology and virus isolation. It was determined that the best time to sacrifice animals for the purpose of virus recovery was between 72 and 96 hours post inoculation. To prepare backpass inoculum, a 50\% (weight per volume) small intestinal tissue extract (sie) was prepared from the remainder of the small intestine. A portion of the extract was then orally fed to the next calf. This procedure was continued until 5-consecutive backpassages in cc-calves had been completed. All extracts were titrated and the amount of virus used in each backpassage inoculum was determined. Virus isolation/titration was performed by inoculating confluent human rectal tumor cells (HRTs) and staining by indirect immunoflourescence at 72 hours post-inoculation. All cccalf tissue samples appeared normal and no signs of villous atrophy were observed in any of the five backpassages. BCV (NCD) was isolated and identified from the small intestinal samples of all of the backpassage inoculated animals, although the amount of virus recovered dropped significantly after the 2 nd backpassage. 
Table 1. Comparison of BCV (NCD) calf backpassage number 5 to virulent NCD challenge

\begin{tabular}{lcc}
\hline Virus inoculation & Clinical observations & Mortality \\
\hline Backpass - 5 & $0 / 5(0 \%)$ & $0 / 5(0 \%)$ \\
NCD Challenge & $5 / 5(100 \%)$ & $3 / 5(60 \%)$ \\
\hline
\end{tabular}

To confirm the safety of the BCV (NCD) vaccine the 5th backpassage sie was used to orally inoculate $5 \mathrm{cc}$-calves. These animals were compared to $5 \mathrm{cc}$-calves that were orally inoculated with a virulent intestinal extract (standard challenge). Animals were between 7 and 10 days of age and were housed in germfree bubbles, fed medicated milk replacer and observed for 14-days post-inoculation. Both viruses were titrated prior to oral inoculation. The virulent virus was diluted so as to have the same titer for inoculation as backpass \#5. All five backpassage \#5 inoculated cc-calves remained clinically normal. Two of the five animals developed a creamy diarrhea that persisted for 3 days, but it was at 10-days after inoculation and this was not considered a clinical sign due to inoculation. Only a non-pilliated beta-hemolytic E. coli was isolated from the fecal samples collected from these calves. All of the backpass \#5 animals significantly gained weight at a greater rate than the virulent inoculated animals. All five virulent inoculated animals developed severe watery diarrhea within 72 hours post-inoculation which persisted for up to 10 days Three of the five virulent-inoculated calves were moribund and had to be sacrificed prior to the end of the test. Autopsy results were typical of a severe bovine coronavirus infection with the villi in the jejunum and ileum appearing to be completely denuded. BCV was isolated from the small intestine of each of the sacrificed animals.

\section{BCV (NCD) Vaccine Protects against a Winter Dysentery Challenge}

Six cc-calves between the ages of 7 to 10 days were used in this study. Three animals were orally inoculated with the BCV (NCD) vaccine and the other three animals remained as nonvaccinated controls. All animals were orally challenged with a WD virulent intestinal extract at 24 days of age and were observed for an additional 14-days. Fecal samples were collected at time of challenge, 3 days post-challenge (3-DPC), 5-DPC, $7-$ DPC, 10-DPC and 14-DPC. The vaccinated animals had normal feces and remained clinically normal and gained weight at a greater rate than the nonvaccinated controls during the post-challenge period. At 72 hours post-challenge all nonvaccinated controls had developed a severe watery diarrhea that persisted for 5 to 7 days. No virus was detected in the vaccinated group post-challenge compared to the control group in which virus was detected in fecal samples from 3-DPC to 10-DPC.

Table 2. Effectiveness of $\mathrm{BCV}(\mathrm{NCD})$ vaccine against a virulent winter dysentery challenge

\begin{tabular}{lcccccc}
\hline & Morbidity & \multicolumn{5}{c}{ Fecal shedding post-challenge (\%) } \\
\cline { 3 - 7 } Group & incidence (\%) & 3-DPC & 5-DPC & 7-DPC & 10-DPC & 14-DPC \\
\hline Vaccinates & 0 & 0 & 0 & 0 & 0 & 0 \\
Controls & 100 & 100 & 100 & 100 & 33 & 0 \\
\hline
\end{tabular}




\section{DISCUSSION}

In conclusion, a vaccine for bovine coronavirus infections has been developed using a newborn calf diarrheal isolate (NCD). The virus was adapted and serially passaged in an established diploid swine testicular cell line. A vaccine candidate was determined in a series of studies where cc-calves were orally inoculated with different cell culture passage levels of BCV (NCD). These various BCV cell culture passaged virus isolates should prove to be useful for determining changes on a molecular level. Master seed has been prepared and passed all testing as outlined by 9 CFR. The vaccine strain master seed remained safe even after 6 backpassages in calves as determined in a side by side study with virulent NCD inoculated calves.

This BCV (NCD) vaccine is unique in that it will prevent both WD and NCD infections. The BCV (NCD) vaccine was able to prevent clinical signs and inhibit virulent virus shedding associated with a severe WD challenge.

This BCV (NCD) vaccine will provide the worldwide cattle industry with means to control prevalent bovine coronavirus infections and thereby satisfy a demand for a more efficacious product in the field.

\section{REFERENCES}

Babiuk, L.A., Sabara, M., and Hudson, G.R., 1985, Rotavirus and coronavirus infections in animals, Prog. Vet. Microbiol. Immunol. 1: 80-120.

Millane, G., Michaud, L., and Dea, S., 1995, Biological and molecular differentiation between coronaviruses associated with neonatal calf diarrhoea and winter dysentery in adult cattle, Adv. Exp. Med. Biol. 380: 29-33.

Saif, L. J., and Theil, K. T. (eds), 1990, Viral diarreheas of man and animals. CRC Press, Boca Raton, Florida. 